\title{
Research Note \\ On the close optical companion of V1494 Aql: Problems with light curve modelling ${ }^{\star}$
}

\author{
L. L. Kiss ${ }^{1, \star \star}$, B. Csák ${ }^{2}$, and A. Derekas ${ }^{1}$ \\ ${ }^{1}$ School of Physics, University of Sydney 2006, Australia \\ 2 Department of Experimental Physics and Astronomical Observatory, University of Szeged, Szeged, Dóm tér 9., 6720 Hungary
}

Received 27 October 2003 / Accepted 9 December 2003

\begin{abstract}
New observations of the eclipsing nova V1494 Aql were analysed to estimate the effect of the optical companion located 1 ." 4 to the southwest. $R$ and $I$-band images (stellar $F W H M \approx 11^{\prime \prime} 2-11^{\prime \prime} 4$ ) taken close to the primary minimum were used to determine relative magnitude differences between the nova and the companion, which were found to be $\Delta R \approx 0.20$ and $\Delta I \approx 0.52$ (in the sense nova minus companion). After correcting $R$-band time-series observations for the secondary light, the eclipse depth has been found to be almost twice as deep. Before modelling any late eclipsing light curve that is available in the literature, the data must be corrected for the light of the companion.
\end{abstract}

Key words. stars: novae, cataclysmic variables - stars: individual: V1494 Aql

\section{Introduction}

V1494 Aql (=Nova Aql 1999/2) is one of the brightest nova outbursts observed in the last decade. Shortly after discovery in December 1999 (Pereira et al. 1999), it peaked at $m_{\mathrm{vis}}=4 \mathrm{mag}$, followed by rapid dimming ( $t_{3}=16 \mathrm{~d}$, Kiss \& Thomson 2000). The light curve showed a classical transition phase with quasiperiodic oscillations (see an updated light curve in Kato et al. 2003). Optical spectroscopy (Kiss \& Thomson 2000; Anupama et al. 2001; Arkhipova et al. 2002; Iijama \& Esenoglu 2003) showed that the nova belonged to the "Fe II" class of Williams (1992) with early expansion velocities up to $2000 \mathrm{~km} \mathrm{~s}^{-1}$. Spectroscopy in the transition phase suggested the presence of high velocity jets, while the MMRD absolute magnitude (Kiss \& Thomson 2000) was combined with spectroscopic reddening determination which placed the star $1.6 \pm 0.2 \mathrm{kpc}$ from the Sun (Iijama \& Esenoglu 2003). Early X-ray observations were reported by Drake et al. (2003) revealing short-period (2523 s) oscillations that were interpreted as pulsations of the white dwarf in the nova. Spectropolarimetric evidence of an asymmetric outburst was presented by Kawabata et al. (2001).

The photometric history is rich in observations. Kato et al. (2003) gave an excellent review of the published works, here

Send offprint requests to: L. L. Kiss,

e-mail: 1aszlo@physics.usyd.edu.au

* Based on data obtained at the Anglo-Australian Observatory and Mount Stromlo and Siding Spring Observatories.

$\star \star$ On leave from University of Szeged, Hungary. we just briefly mention the main results. After the discovery of short-period modulations of the light curve (Novak et al. 2000), Retter et al. (2000) pointed out the double-wave nature of the modulation with a peak-to-peak amplitude of $0.07,8$ months after the outburst. Bos et al. (2001) observed a strong amplitude increase of the modulation and suggested the eclipsing nature of the system. Barsukova \& Goranskii (2003) refined the orbital period, giving $P_{\text {orb }}=0.1346141(5) \mathrm{d}$. Pavlenko et al. (2003) made a multicolour study of the star concluding that the light curve shape might be explained by a self eclipsing accretion column in the magnetic variable accompanied by partial eclipses of the accretion region by the secondary component.

We have been following the star since its discovery, mostly monitoring it spectroscopically (Kiss \& Thomson 2000; Kiss et al. in preparation). Here we report on new imaging and spectroscopy carried out in 2003, which revealed the presence of a close optical companion (already reported by Barsukova \& Goranskii 2003, however, we were not aware of this before making the observations). Interestingly, none of the recent studies tried to correct for the photometric effects of the companion. We point out the necessity of such a correction.

\section{Observations and data reduction}

We discuss three different datasets. On May 10, 2003, between HJD = 2452 770.148-2 452 770.317, four hours of service observations were carried out using the $3.9 \mathrm{~m}$ Anglo-Australian Telescope (AAT) equipped with the RGO spectrograph. 

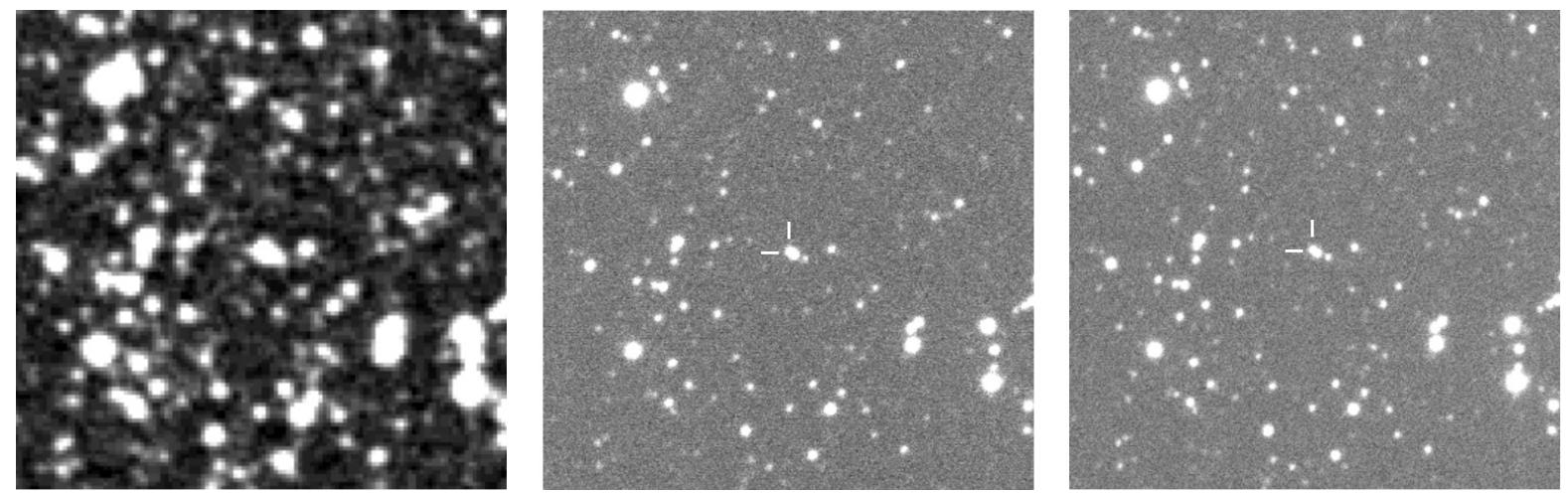

Fig. 1. $120^{\prime \prime} \times 120^{\prime \prime}$ fields centered on V1494 Aql. Left to right: POSS2 red, $R$-band image at HJD $=2452930.9568$ (stellar $F W H M=1{ }^{\prime \prime} 4$ ), $I$-band image at $\mathrm{HJD}=2452930.9526\left(F W H M=1{ }^{\prime \prime} 2\right)$. Dashes point to the nova, identified through the eclipses. North is up, east is to the left.

We obtained 41 medium-resolution spectra (exposure time 300 s) covering 5800-7300 A, with typical $S / N$ ratios of 50 $(\lambda / \Delta \lambda=6000)$. One flux standard and one smooth-spectrum standard star were also observed. The seeing on that night was about 1 .'3, as judged from the width of the standard spectra. The position angle of the 2 -arcsec wide slit was constant at $200^{\circ}$.

We then made time-series $R$-band observations with the $1.0 \mathrm{~m}$ telescope of the Australian National University in Siding Spring on three consecutive nights in August 2003 (6-8th), with a total time span of $\sim 12 \mathrm{~h}$. The detector was the middle $1 \mathrm{k} \times 1 \mathrm{k}$ subframe of one of the eight $2 \mathrm{k} \times 4 \mathrm{k}$ chips of the Wide Field Imager, giving 6.5 $\times 6.5$ field of view (this corresponds to $0.38 /$ pixel image scale). The exposure time was 180 s. Since V1494 Aql was a secondary target for bright and medium to bad seeing nights (the primary program addressed globular clusters), these time-series data were obtained under 2.5 to 5 arcsec seeings.

The last dataset consists of eight CCD images ( 4 in $R$ and 4 in $I$ ) taken on Oct. 18, 2003. The instrument was the same as in August 2003. The seeing varied between $1^{\prime \prime} 2-11^{\prime \prime}$, which was the best experienced in a three-week long run. The exposure time was only $30 \mathrm{~s}$ to get as sharp an image as possible. We noticed the optical companion of V1494 Aql and that initiated a careful re-check of the literature and previous data.

All observations were reduced with $\mathrm{IRAF}^{1}$ in standard fashion. Spectroscopic reductions included bias, flat and sky background corrections. Aperture extraction and wavelength calibrations were done with the task doslit utilizing $\mathrm{CuAr}$ spectral lamp exposures taken before and after every ten stellar exposures. The flux calibration used a spectrum of LTT 7379, a G0-type spectroscopic standard. Time-series direct images were corrected with bias and sky-flat frames, while instrumental $R$-band magnitudes were calculated with simple aperture photometry (the diameter of the aperture was set to 6.'5) with respect to comparison stars located within 1'.0. Following Barsukova \& Goranskii (2003), the main

${ }^{1}$ IRAF is distributed by the National Optical Astronomy Observatories, which are operated by the Association of Universities for Research in Astronomy, Inc., under cooperative agreement with the National Science Foundation. comparison was GSC 0473-4227. The typical photometric accuracy was $\pm 0.01-0$. 02 .

\section{Discussion}

\subsection{Astrometry}

The original purpose of making sharp images on October 18, 2003 was to check for the presence of a resolvable nova shell. The predicted angular radius of the shell 3.8 years after the outburst is 0.'94 (following Kiss \& Thomson 2000 and adopting the distance revised by Iijama \& Esenoglu 2003). Instead of a shell, we found two closely separated stars within $\pm 1^{\prime \prime}$. In Fig. 1 we show our best CCD frames compared to the POSS2 red image (this is the sharpest POSS2 image). It is quite obvious that the 8 -shaped profile is caused by two stars close to the limit of resolution. The POSS2 image has too low resolution and is too crowded to recognize this. None of the available POSS, POSS2 and 2MASS images of the field shows the nova and its companion resolved, so that our first conclusion is that we do not have any direct information on the progenitor.

The next step was the identification of the eclipsing component. For this, we used our time-series observations taken 2 months earlier. Even the best images were made under 2'.5 seeing, which prevented earlier recognition of the pair. Image differences, however, showed that the eclipsing variable is the eastern component, as concluded by Barsukova \& Goranskii (2003).

Although the chance of having a nova and an eclipsing binary within $2^{\prime \prime}$ seems to be very close to zero (the colours reported by Barsukova \& Goranskii (2003) did not support this either), we made an astrometric solution of the best $I$-band image and compared the coordinates to the published values. We chose 21 non-saturated stars within $\pm 1^{\prime}$ of the nova and took their J2000.0 coordinates from the GSC2. 2 catalog. Pixel coordinates were determined by psf-fitting (with the daophot package in IRAF). The residuals of the solution were \pm 0 !' 2 , which we adopted as the astrometric uncertainty. The resulting coordinates are shown in Table 1. We plot them together with the coordinates of the nova and assumed progenitor (Pereira et al. 1999 ) in Fig. 2. According to this, the separation and the position angle of the companion are $1^{\prime \prime} .4$ and $230^{\circ}$, respectively. 


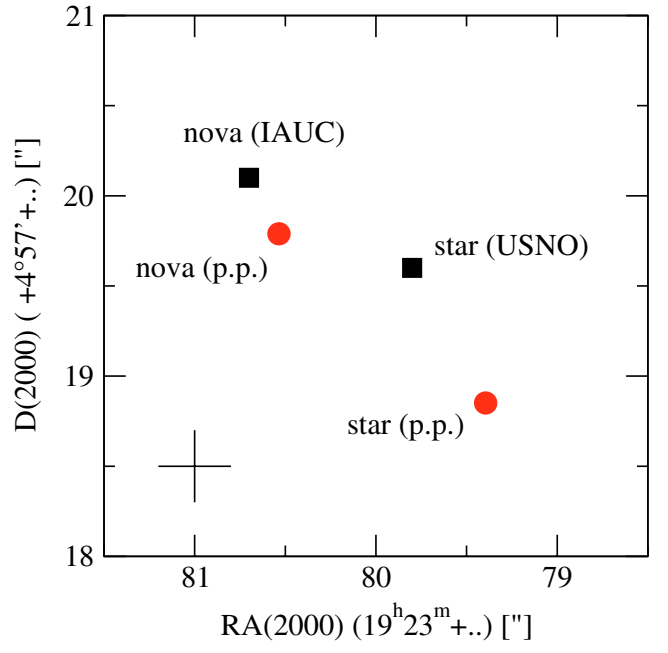

Fig. 2. A comparison of the new astrometric solution and published coordinates of the nova and the suspected progenitor (Pereira et al. 1999). The cross in the left corner shows the uncertainty.

We have two conclusions based on Fig. 2. Firstly, the positional agreement between the eclipsing star and the nova coordinate measured by di Cicco (Pereira et al. 1999) shows that the nova and the eclipsing binary do indeed coincide. Secondly, the pre-outburst USNO position falls almost exactly halfway between the nova and the companion. We interpret this as indirect evidence of the similar apparent brightnesses of the progenitor and the companion.

\subsection{Photometry}

It is evident from Fig. 1 that the nova is fainter than the companion both in $R$ and $I$ (by chance, the analysed images were taken very close to the primary eclipse; the ephemeris in Kato et al. 2003 gives $\phi(R)=0.035$ and $\phi(I)=0.002)$. This means that the estimated contribution of the companion is much larger than quoted in Barsukova \& Goranskii (2003) $(\sim 34 \%$ in $R$ ) and it may present a significant problem when modelling the light curve. To quantify it, we have measured relative magnitudes with respect to GSC 0473-4227 ( $R=12^{\mathrm{m}} \cdot 94, I=12 \mathrm{~m}$. 05 , Barsukova \& Goranskii 2003) and corrected the light curve accordingly. Neglecting colour-dependent transformation terms (being of the order of a few hundredth mag for a wide range of colour differences, Sung \& Bessell 2000), the resulting magnitudes (Table 1) revealed that the nova was fainter by 0.20 in $R$ and by 0.52 in $I$. The error budget of these values consists of the individual psf-fitting errors calculated by the task allstar $(0.05$ for the nova and the companion and 0.006 for the comparison), the uncertainty of the standard magnitudes of the comparison $(0.02)$ and the neglected colour transformation terms (we assumed $0 \mathrm{~m}$ 02). Consequently, the final uncertainty is about 0.06 (it is marginally better in $I$ thanks to the better resolution).

We illustrate the secondary light correction in Fig. 3 (the companion's magnitude was transformed to flux and was subtracted from the flux values of the light curve data; the results were transformed back to magnitudes). The middle curve in
Table 1. Astrometry and photometry of the nova and the companion.

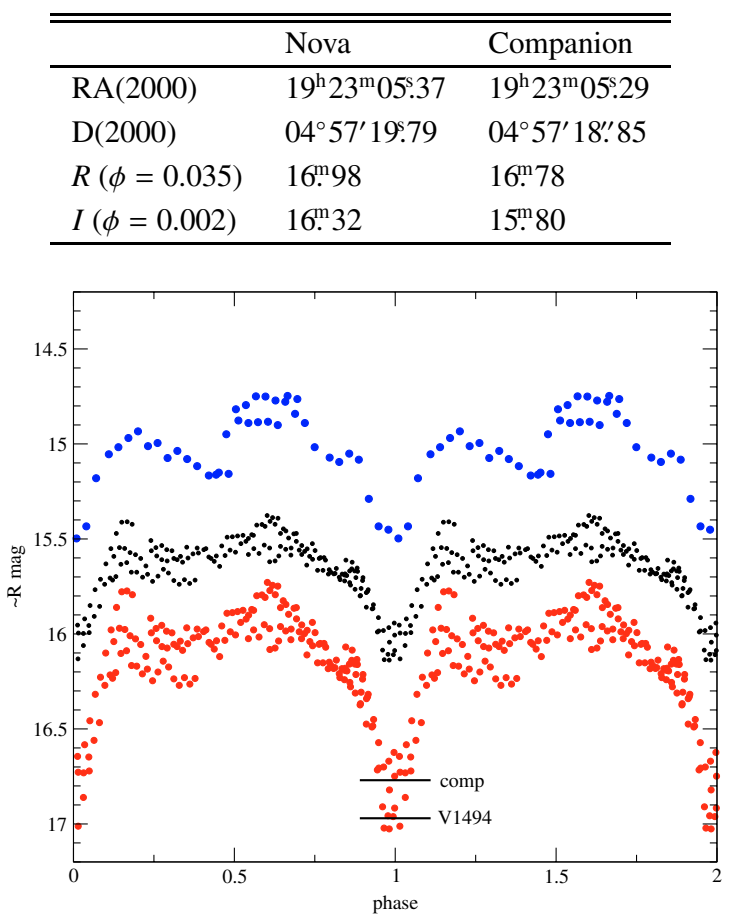

Fig. 3. Eclipsing light curves. Upper curve: integrated magnitudes calculated from the flux-calibrated AAT spectra; middle curve: phase diagram of all data taken in August, 2003; lower curve: the same after correcting for the secondary light.

Fig. 3 shows the phased $R$-band data (using the ephemeris in Kato et al. 2003: $\mathrm{BJD}_{\min }=2452458.3230+0.1346138 \times E$ ), while the corrected curve is the bottom one. The eclipse depth (defined as the brightness difference between $\phi=0$ and $\phi=$ 0.15 ) increased by a factor of two from $\sim 00^{\mathrm{m}} 6$ to $\sim 1 \mathrm{~m} 2$, which is obviously not a negligible effect. It is quite surprising that none of the recent studies applied this correction. On one hand, both Barsukova \& Goranskii (2003) and Pavlenko et al. (2003) mentioned the presence of the companion but they did not go beyond that. On the other hand, despite using unfiltered observations heavily influenced by the red companion, Kato et al. (2003) did not even mention the problem. Our conclusion is that one has to be very careful when analysing observations of V1494 Aql and reliable modelling requires accurate correction for the light of the companion.

\subsection{Spectroscopy}

Further details on the companion's nature are provided by the AAT spectroscopy. A sample spectrum is shown in the upper panel of Fig. 4. Beside a flat continuum, the spectrum is dominated by the complex $\mathrm{H} \alpha$ line and an emission blend at $6080 \AA$. Weak absorption lines are present in the blue and the red third of the spectrum, of which the red one mostly contains atmospheric telluric lines. In the blue part the sodium D is the strongest absorption. The integrated fluxes converted to magnitudes draw the same eclipsing light curve as the CCD observations do (see the top curve in Fig. 3). After recognizing the 

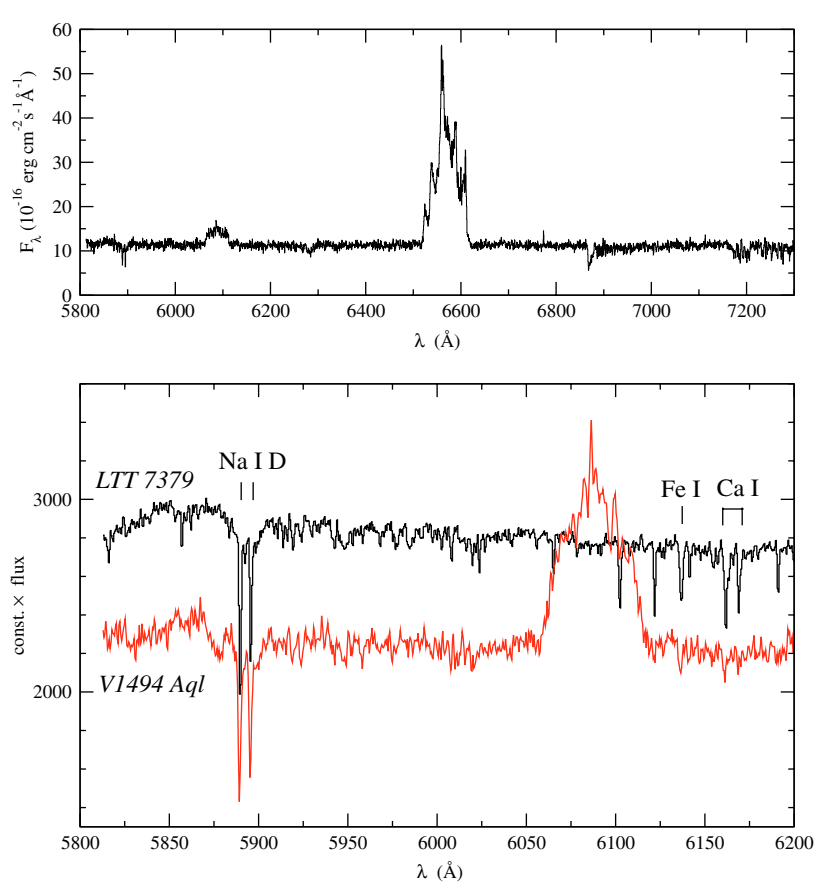

Fig. 4. Top panel: a sample AAT-spectrum of V1494 Aql. Note the symmetric structure of the $\mathrm{H} \alpha$ profile. Bottom panel: a close-up view of the bluest quarter compared to the flux-standard.

problems with the companion, we checked the original spectrum images and found that nova spectra were indeed wider than the standard ones (but not wide enough for deblending). That means we had the same contamination from the companion as in the case of the imaging.

The absence of molecular bands in the spectrum exclude the possibility of a late-type companion. Furthermore, we could identify a number of common absorption lines in the spectra of the nova and the G0-type flux standard. The most prominent ones are marked in the bottom panel of Fig. 4. The resemblance suggests the spectral type of the companion is similar to the flux standard, most likely between late $\mathrm{F}$ and early $\mathrm{G}$.

Finally, the sodium D line has some interesting properties. Iijama \& Esenoglu (2003) detected strong interstellar sodium $D$ lines three months after the outburst. They measured the following equivalent widths: $E W_{\mathrm{D} 1}=0.46 \pm 0.01 \AA$ and $E W_{\mathrm{D} 2}=$ $0.55 \pm 0.01 \AA$. As a comparison, the blended AAT spectra result in $E W_{\mathrm{D} 1}=0.50 \pm 0.02 \AA$ and $E W_{\mathrm{D} 2}=0.65 \pm 0.02 \AA$, with possible greater systematic uncertainty due to the ambiguous continuum level. The larger equivalent widths are consistent with the assumption that we see a sum of stellar and interstellar absorptions. Since we do not detect any additional line doubling of the doublet (within $\pm 5 \mathrm{~km} \mathrm{~s}^{-1}$ ), either the $\sim$ G-type companion has similar radial velocity as the cloud in which the interstellar component, detected by Iijama \& Esenoglu (2003), originated or the continuum we see in Fig. 4 mostly comes from the reddened companion (in which case the radial velocities of the companion and the cloud still have to agree unless the cloud is farther away).

\section{Conclusions}

V1494 Aql is an intriguing eclipsing nova that has initiated recently a number of independent photometric and spectroscopic studies. None of them has tried to correct for the effects of the close companion. With the presented properties of the star, this neglect can be dangerous and has the potential to lead to unreliable light curve models. The red and far red spectral regions are especially affected by the companion, which is a major obstacle to using unfiltered CCD measurements.

Based on the results presented in this paper, we can summarize our knowledge on the companion of V1494 Aql. It is located 1".4 SW of the eclipsing nova. At minimum, the nova is fainter by 0.2 in $R$ and 0.52 in $I$, while at maximum, it is brighter by about $0 .{ }^{\mathrm{m}} 6-0 \mathrm{~m} \cdot 8$ in $R$, depending on the highly variable light curve shape (see Pavlenko et al. 2003 and the scatter of the phase diagrams in Fig. 3). A tentative spectral type has been assigned to the companion based on medium-resolution spectra with the AAT.

Adopting $E(B-V)=0 \mathrm{~m} \cdot 6, d=1.6 \mathrm{kpc}$ and assuming the mean corrected $R$ magnitude ( $\sim 16.3)$ of the nova is close to the quiescent brightness, the progenitor's absolute magnitude was about $M_{R}=3.9$, which leaves unchanged our conclusion (Kiss \& Thomson 2000). However, further highresolution imaging, preferably made under sub-arcsecond seeing, is needed to allow accurate photometric corrections and light curve modelling.

Acknowledgements. This work has been supported by the FKFP Grant 0010/2001, OTKA Grants \#F043203 and \#T034615 and the Australian Research Council. We are grateful to the TACs of the Anglo-Australian Observatory and the Mount Stromlo and Siding Spring Observatories for allocating telescope time used for this study. We also wish to thank the assistance of Péter Székely during the timeseries photometric observations. The NASA ADS Abstract Service was used to access data and references.

\section{References}

Anupama, G. C., Sahu, D. K., \& Mayya, Y. D. 2001, Bull. Astron. Soc. India, 29, 375

Arkhipova, V. P., Burlak, M. A., \& Esipov, V. F. 2002, Astron. Lett., 28,100

Barsukova, E. A., \& Goranskii, V. P. 2003, Astron. Lett., 29, 195

Bos, M., Retter, A., Cook, L., \& Novak, R. 2001, IAU Circ., 7665

Drake, J. J., Wagner, R. M., Starrfield, S., et al. 2003, ApJ, 584, 448

Iijama, T., \& Esenoglu, H. H. 2003, A\&A, 404, 997

Kato, T., Ishioka, R., Uemura, M., Starkey, D. R., \& Krajci, T. 2003, PASJ, in press [astro-ph/0310519]

Kawabata, K. S., Akitaya, H., Hirakata, N., et al. 2001, ApJ, 552, 782

Kiss L. L., \& Thomson, J. R. 2000, A\&A, 355, L9

Novak, R., Retter, A., Cook, L., Korcakova, D., \& Janik, J. 2000, IAU Circ., 7448

Pavlenko, E. P., Dudka, O. I., \& Baklanov, A. V. 2003, Astrophysics, 46, 191

Pereira, A., di Cicco, D., Vitorino, C., \& Green, D. W. E. 1999, IAU Circ., 7323

Retter, A., Cook, L., Novak, R., et al. 2000, IAU Circ., 7537

Sung, H.,\& Bessell, M. S. 2000, PASA, 17, 244

Williams, R. E. 1992, AJ, 104, 725 